\title{
Características sociodemográficas e nutricionais de crianças brasileiras menores de 2 anos beneficiárias de programas de transferência condicionada de renda em 2006
}

\author{
Socio-demographic and nutritional characteristics \\ of Brazilian children under 2 years of age who were beneficiaries \\ of conditional cash transfer programs in 2006
}

Juliana Bergamo Vega ${ }^{1}$

José Augusto de Aguiar Carrazedo Taddei ${ }^{1}$

Ana Paula Poblacion ${ }^{1}$

${ }^{1}$ Disciplina de Nutrologia, Departamento de Pediatria, Universidade Federal de São Paulo. Rua Loefgren 1647, Vila Clementino.

04.040-032 São Paulo SP Brasil.

jvbergamo@hotmail.com
Abstract The conditional cash transfer programs (PTCR) seek to combat poverty and hunger and to ensure food security. Children under the age of two of beneficiary families are more vulnerable to environmental conditions. This study seeks to describe this population according to socio-econom$i c$, demographic and nutritional variables. Secondary data from the National Survey on Demography and Health of Women and Children (PNDS-2006) were used. Data were described using mean and standard deviations. Associations were described using the chi-square test with confidence intervals of 95\%. Of the 1735 children, $29.4 \%$ were PCTR beneficiaries with average family income of $R \$ 430.20$ and average benefits of $R \$ 75$. Among those living in the Northeast or in the rural area of the country, one in every three received benefits. Among those experiencing severe food insecurity, 52.3\% were PTCR beneficiaries. Children from socio-economic classes $D$ and $E$ and whose mothers had less than four years education were associated with PTCR. Among the nutritional disorders of child beneficiaries, overweight for age prevailed. Of those who ceased exclusive breastfeeding in less than two months, $31.2 \%$ were beneficiaries. The need for preventive nutritional education was identified in order to optimize the use of benefits.

Key words Public policies, Poverty, Food and nutrition security, Nutrition assessment
Resumo Programas de transferência condicionada de renda (PTCR) visam o combate à pobreza e à fome e a garantia da segurança alimentar. Crianças menores de 2 anos de famílias beneficiárias são mais vulneráveis às condições ambientais. Este estudo objetiva caracterizar esta população segundo variáveis socioeconômicas, demográficas e nutricionais. Utilizaram-se dados secundários da Pesquisa Nacional de Demografia e Saúde da Criança e da Mulher (PNDS-2006). Descreveramse os dados em média e desvios-padrão. Para as associações utilizou-se o teste qui-quadrado e intervalos de confiança de 95\%. Das 1735 crianças 29,4\% eram beneficiárias de PTCR com renda média familiar de $R \$ 430,20$ e valor médio dos benefícios de $R \$ 75$. Dentre as que residiam no Nordeste ou na região rural do país, uma em cada três recebiam PTCR. Entre as que viviam em insegurança alimentar grave, 52,3\% pertenciam a PTCR. Classes econômicas D e E e baixa escolaridade materna associaram-se a PTCR. Entre os desvios nutricionais das crianças beneficiárias, prevaleceu $o$ peso elevado para idade. Das que interromperamo aleitamento materno exclusivo antes dos dois meses, 31,2\% eram de PTCR. Identifica-se a necessidade de educação nutricional preventiva a fim de otimizar a utilização do benefício.

Palavras-chave Políticas públicas, Pobreza, Segurança alimentar e nutricional, Avaliação nutricional 


\section{Introdução}

A assistência social acompanha o desenvolvimento do capitalismo, haja vista que este promove a desigualdade entre as classes, trazendo como consequência, para grande parte da população limitado acesso às diversas oportunidades ${ }^{1,2}$. $\mathrm{A}$ influência das desigualdades sociais na saúde humana vem sendo amplamente discutida no campo da saúde coletiva. É assunto de grande relevância para um país como o Brasil, que possui grande parte da população empobrecida, apesar do elevado PIB per capita ${ }^{1,3}$.

A pobreza pode ser compreendida como situação de falência do indivíduo, sendo múltiplas as situações de vulnerabilidade ${ }^{4}$. $\mathrm{Na}$ dinâmica capitalista, a renda determina a capacidade do indivíduo em garantir suas necessidades básicas. No Brasil, apesar da pobreza persistir, esta vem apresentando tendência de queda nos últimos $a \operatorname{sos}^{5}$. Esta mudança acompanha as transformações que as políticas de proteção social vêm sofrendo ao longo da história do país.

O principal desafio destas políticas é o de garantir para todos os cidadãos os direitos sociais básicos, reconhecendo-os como um direito humano isento de caráter filantrópico. Existiram programas no Brasil, como o de distribuição de cestas básicas de alimentos ${ }^{6}$, com o intuito de aliviar o problema da fome, mas sem uma estratégia para a resolução do problema das desigualdades sociais. Desde a década de noventa, os programas de transferência condicionada de renda (PTCR) vêm sendo a opção do governo federal para substituir ou complementar as demais intervenções como esta em um processo crescente de cobertura ${ }^{4}$. Tais programas ocupam lugar de destaque nas agendas das políticas públicas no Brasil discutindo o combate à pobreza, a promoção da saúde e a segurança alimentar e nutricional das populações, integrada com ações que promovam a redistribuição de riquezas de forma mais democrática ${ }^{4,6,7}$.

Vários programas foram criados por ministérios e secretarias diferentes, mas a falta de uma gestão centralizada resultou em pouca efetividade e alto custo operacional ${ }^{6-8}$. A fim de otimizar os recursos foi criado em 2003 o Programa Bolsa Família (PBF), que unificou os programas federais Bolsa Alimentação, Bolsa Escola e Auxílio Gás. Como parte do programa Fome Zero, é hoje considerado o maior programa de transferência de renda do mundo ${ }^{4,6-8}$. Tem como objetivo atender brasileiros com renda familiar per capita inferior a $\mathrm{R} \$ 70,00$ (US\$35) mensais. Atu- almente cobre mais de 13 milhões de famílias, ou seja, mais de 40 milhões de pessoas em todo o território nacional. O benefício varia de acordo com as características de cada família considerando a renda mensal familiar per capita, número de crianças e adolescentes com idade inferior a 17 anos, gestantes e nutrizes. $\mathrm{O}$ valor acumulado do repasse financeiro de janeiro a setembro de 2012 foi de 15,3 bilhões de reais, equivalentes a 7,6 bilhões de dólares 9 .

O PBF tem por objetivo combater a fome, a pobreza e as desigualdades sociais por meio da transferência de renda, associado à garantia do acesso aos direitos sociais básicos como a saúde, a educação, a assistência social e a segurança alimentar, além de objetivar a promoção da inclusão social pela construção de meios para que as famílias do programa saiam da situação de vulnerabilidade em que se encontram ${ }^{8}$.

Algumas condicionalidades são exigidas dos beneficiários como o acompanhamento pré-natal da gestante, a frequência escolar das crianças e o monitoramento, por meio da rede pública de saúde, do crescimento, do desenvolvimento infantil e do calendário de vacinação ${ }^{8}$. O cumprimento dessas exigências pressupõe que o indivíduo beneficiário possa melhorar suas condições gerais de educação e saúde, incluindo também as condições nutricionais. Acredita-se que o incremento na renda poderá promover a melhora do estado nutricional dos indivíduos beneficiários, já que os estratos inferiores de renda têm dificuldade de acesso a alimentos em qualidade e quantidade suficientes para garantir a segurança alimentar e nutricional ${ }^{10}$. Espera-se então que todas estas ações possam causar impacto significativo na vida dos beneficiários, principalmente entre as crianças, a fim de romper o ciclo da pobreza e possibilitar oportunidades de mudança também para as novas gerações.

No entanto, o desafio dos PTCR em atingir seus objetivos se defronta com alguns obstáculos, haja vista que a pobreza é um fenômeno complexo que requer a compreensão da influência que sofre das dimensões econômica, social, cultural, histórica e política. É um fenômeno multidimensional, não se restringindo apenas à insuficiência de renda ${ }^{8}$.

Existem vários debates em torno dos PTCR. São discussões acerca da efetividade das condicionalidades e da legitimidade das suas exigências, visto que são direitos incondicionais garantidos a todos, os erros de inclusão com beneficiários que tem renda acima do limite de corte do programa e a exclusão de pessoas que cumprem os 
critérios de elegibilidade do programa, mas não são beneficiadas ${ }^{8,11}$.

A fim de contribuir para a compreensão de algumas questões que envolvem os programas de transferência condicionada de renda, este trabalho tem por objetivo caracterizar crianças menores de 2 anos beneficiárias de qualquer PTCR vigente no Brasil em 2006, com destaque ao PBF, quanto a variáveis demográficas e socioeconômicas, bem como avaliar algumas variáveis nutricionais, comparando-as com a população não beneficiária, explorando os níveis de cumprimento dos objetivos almejados pelos programas.

\section{Métodos}

Trata-se de um estudo transversal, realizado a partir de dados secundários da Pesquisa Nacional de Demografia e Saúde da Criança e da $\mathrm{Mu}$ lher (PNDS), realizada no ano $2006^{12}$. Esta pesquisa faz parte da quinta fase do MEASURE DHS (Demographic and Health Survey), um projeto de investigação global de indicadores de saúde e nutrição de mulheres e crianças nos países em desenvolvimento. No Brasil, o estudo de 2006 é a sua terceira edição, tendo sido realizada também em 1986 e $1996^{13}$.

A PNDS caracteriza-se por ser um inquérito domiciliar, por amostragem probabilística complexa, com representatividade nacional. As unidades amostrais foram selecionadas em dois estágios: o primeiro com as unidades primárias compostas por setores censitários, e o segundo pelas unidades secundárias formadas por todos os domicílios dos setores sorteados. Foram considerados para o universo do estudo, domicílios particulares, em setores comuns ou não especiais, incluindo favelas, selecionados em dez estratos amostrais, que compõem uma combinação das cinco grandes regiões geográficas brasileiras, com as áreas urbanas e rurais ${ }^{13}$. Foram coletados os dados de 15.575 mulheres em idade reprodutiva, de 15 a 49 anos de idade, e aproximadamente 5.000 crianças menores de cinco anos, sendo as informações obtidas por intermédio de dois questionários, apresentação de cartões de vacinação e mensurações antropométricas de mulheres e crianças, além de amostras sanguíneas de vitamina A e ferro, e o teor de sódio do sal de cozinha dos domicílios ${ }^{13}$.

Para este trabalho foi considerada uma subamostra de 1.735 crianças. Como critério de inclusão foram selecionados crianças com idade inferior a dois anos, residir com suas mães na ocasião do inquérito e conter informações antropométricas. A escolha da faixa etária foi determinada pela forte influência dos fatores ambientais sobre seu estado de saúde e nutrição ${ }^{14,15}$.

\section{Fontes de renda}

A descrição das fontes de renda dos domicílios foi realizada por meio da análise da média e do desvio-padrão das variáveis: Rendimento total do domicílio proveniente de trabalho (salários); Seguro-desemprego; Aposentadoria; Pensão Alimentícia; Outra pensão; Renda Mensal Vitalícia; Rendimento de Aluguel de imóvel; Doações; Renda proveniente de vendas de produtos e Programas de transferência condicionada de renda.

Como para a análise das fontes de renda a unidade de estudo é o domicílio, foi necessário considerar somente uma criança menor de 2 anos por residência, para que a representação daquela unidade amostral não viesse a ser duplicada na análise dos dados. Sendo assim, quando uma mesma mulher da amostra tinha mais do que um filho menor de 2 anos, foi considerado somente o de maior idade. Quando havia duas ou mais crianças menores de 2 anos, filhos de mães diferentes, residentes no mesmo domicílio, foram excluídas as crianças mais novas. Com o mesmo objetivo, nas situações em que existiam gemelares, selecionou-se o gêmeo que havia nascido em melhores condições. No primeiro passo considerou-se apenas o de maior peso ao nascimento anotado no cartão da criança. Caso não existisse essa informação foi selecionado o de maior peso ao nascer referido pela mãe. Por fim, caso não houvesse ambas as informações considerou-se apenas o primeiro listado no número de ordem do filho nascido vivo $(\mathrm{n}=76)$.

\section{Programas de transferência condicionada de renda (PTCR)}

Foi criada a variável "Programa de transferência condicionada de renda" (PTCR) que identifica as crianças menores de 2 anos que pertenciam a famílias que recebiam benefício de, ao menos, um dos programas de transferência condicionada de renda vigentes em 2006: Programa Bolsa Escola, Benefício de prestador de contas (BPC), Auxílio Gás, Cartão Alimentação, Programa de Erradicação do Trabalho Infantil (PETI), Programa Agente Jovem e Programa Bolsa Família. Vale ressaltar que em 2006 os PTCR ainda estavam em processo de unificação. Sendo assim, algumas famílias estavam cadastradas em um ou mais 
PTCR, que não somente o PBF. Esta variável foi considerada como dependente para as análises que motivam esta pesquisa.

\section{Variáveis independentes}

As estimativas de prevalências são de representatividade nacional, sendo variáveis independentes do presente estudo, a situação do domicílio (urbano e rural) e as macrorregiões brasileiras (Norte, Nordeste, Sul, Sudeste e Centro-Oeste). Para estas variáveis as estimativas foram calculadas considerando o fator de expansão da amostra.

Também foram consideradas as variáveis independentes: a insegurança alimentar (leve, moderada e grave), as classes econômicas (A, B, C, $\mathrm{D}$ e E), a escolaridade materna (anos de estudo), o estado nutricional pelo índice de peso para idade (baixo peso para idade, eutrófico e peso elevado para idade) e o tempo de aleitamento materno exclusivo (em meses).

\section{Variáveis socioeconômicas}

e Segurança alimentar e nutricional (SAN)

Para a investigação da insegurança alimentar foi utilizada a Escala Brasileira de Insegurança Alimentar (EBIA) ${ }^{16,17}$.

Os domicílios que se encontram em segurança alimentar (SA) são aqueles em que todas as pessoas que residem no domicílio têm acesso regular e permanente a alimentos de qualidade, em quantidade suficiente, sem comprometer o acesso a outras necessidades essenciais, respeitando a diversidade. Os domicílios que se encontram em insegurança alimentar leve (IAL) são caracterizados pelo comprometimento da qualidade da alimentação, preservando a quantidade, tida como adequada para cada um dos moradores do domicílio. Já a insegurança alimentar moderada (IAM), remete a restrições na quantidade ou mudança nos padrões usuais da alimentação, apenas entre os adultos. Os domicílios que apresentam insegurança alimentar grave (IAG) são aqueles que experimentam a quebra do padrão usual da alimentação, com comprometimento da qualidade e redução da quantidade de alimentos nas refeições, de todos os membros da família, inclusive das crianças residentes nesse domicílio ${ }^{16,17}$.

\section{Nível econômico da família}

Para estimar a capacidade de consumo de produtos e serviços utilizou-se o sistema de pon- tuação padronizado do Critério de Classificação Econômica Brasil (CCEB), versão 2008, que quantifica oito bens duráveis do domicílio e o número de empregados mensalistas, além do grau de instrução do chefe da família, gerando pontos de corte para a determinação do poder aquisitivo da pessoa ou da família. Quanto maior a pontuação, maior o poder de compra. A partir de tais informações, estima-se a condição econômica para o domicílio. Para este estudo agregaram-se em cinco (A, B, C, D e E) os oito estratos sugeridos na proposta original dos pontos de corte da Associação Brasileira de Empresas de Pesquisa $(2007 / 2008)^{18}$.

\section{Escolaridade materna}

A variável escolaridade materna foi categorizada em três níveis, 0-4, 5-8 e 9 ou mais anos de estudo, a fim de expressar o grupo de pessoas com baixo nível de alfabetização, alfabetização completa e níveis superiores de escolaridade, respectivamente.

\section{Estado nutricional}

Segundo a metodologia da PNDS, a medida de peso foi obtida em balança eletrônica com precisão de $100 \mathrm{~g}$, calibrada diariamente, no início e no final do dia de trabalho. Foram feitas duas medidas para cada criança para a obtenção da média de peso.

Para classificação do estado nutricional foi utilizado o indicador de peso para idade $(\mathrm{P} / \mathrm{I})$, expresso em escore Z. Foi considerado "baixo peso para idade" valores $<-2$ escore $Z$, "peso adequado para idade", valores entre $\geq-2$ e $\leq+2$ escore Z, e "peso elevado para idade", valores $>$ +2 escore Z. Os valores observados na amostra de \pm 6 escores $Z$ foram considerados desvios acentuados e excluídos das análises ${ }^{19}$.

\section{Tempo de Aleitamento materno exclusivo} (AME)

Foi considerado AME quando a criança era alimentada apenas com leite humano, diretamente do peito ou ordenhado, caracterizado pela a ausência de oferta de líquidos, semissólidos e sólidos, constantes do recordatório simplificado sobre o consumo alimentar de 24 horas que antecederam a entrevista ${ }^{20}$. Esta variável foi estratificada em três categorias, sendo 0-2, 2-4 e 4-6 meses de idade. 
dos significantes os testes que apresentaram probabilidade menor que 5\% ( $\mathrm{p}<0,05)$. Os parâmetros e os respectivos intervalos de confiança de $95 \%$ foram estimados para os dados expandidos, considerando o efeito do desenho amostral. Todas as análises foram realizadas no pacote estatístico Stata/IC 10.0 (StataCorp LP, CollegeStation, TX, USA).

\section{Aspectos éticos}

Os dados da PNDS foram coletados segundo os critérios estabelecidos pela Declaração de Helsinki, tendo todos os procedimentos envolvendo seres humanos sido aprovados pelo Comitê de Ética do Centro de Referência e Treinamento em DST/AIDS, do Departamento de Saúde do Estado de São Paulo.

\section{Resultados e discussão}

A pobreza no Brasil é fruto da extrema desigualdade social, associado à alta concentração de ren$\mathrm{da}^{8,21}$. A disparidade existente entre as classes so-

ciais brasileiras é o foco de políticas públicas como os PTCR, que têm por objetivo minimizar as consequências históricas do desenvolvimento econômico desigual do país e promover a mobilidade social entre os cidadãos da população mais vulnerável. Apesar dos esforços do governo e da sociedade civil em diminuir estes abismos sociais e os dados das pesquisas nacionais apontarem para uma tendência de queda nos últimos anos, a desigualdade de renda brasileira ainda está entre as doze mais altas do mundo ${ }^{22}$.

Tais programas cumprem o objetivo de beneficiar, preponderantemente, aqueles em situação de pobreza e de extrema pobreza, visto que a renda média dos domicílios que recebiam PTCR era de $\mathrm{R} \$ 430,20$ (dados não apresentados em tabela), valor $23 \%$ maior que o salário mínimo vigente em $2006^{23}$.

De acordo com a Tabela 1, o valor médio do benefício recebido era de $\mathrm{R} \$ 74,94(\mathrm{DP} \pm \mathrm{R} \$$ $46,08)$, representando $17,4 \%$ do rendimento total proveniente de salários dos domicílios beneficiados. Entre os domicílios que recebiam benefícios do PBF, a média do valor era de $\mathrm{R} \$ 70,42$ $(\mathrm{DP} \pm \mathrm{R} \$ 27,31)$.

Para dimensionar o poder de compra do benefício no ano de 2006, o valor da cesta básica de alimentos no Estado de São Paulo no mês de dezembro, segundo a Fundação de Proteção e Defesa do Consumidor PROCON, era de R\$ $171,21^{24}$, ou seja, $\mathrm{R} \$ 96,27$ mais caro que o valor médio recebido pelos programas, confirmando

Tabela 1. Distribuição das fontes de renda, em reais (R\$), dos domicílios com crianças menores de 2 anos, Pesquisa Nacional de Demografia e Saúde (PNDS), Brasil, 2006. $(\mathrm{n}=1.659)$

\begin{tabular}{|c|c|c|c|c|c|}
\hline Fonte de renda & $\mathbf{n}^{\mathrm{a}}$ & $\begin{array}{l}\text { Não recebimento } \\
\text { da renda } \\
\text { n }(\%)\end{array}$ & $\begin{array}{c}\text { Recebimento } \\
\text { da renda } \\
\text { n }(\%)\end{array}$ & $\begin{array}{c}\text { Valor médio } \\
\text { da renda } \\
\quad(\mathrm{R} \$)\end{array}$ & $\begin{array}{c}\text { Desvio- } \\
\text { padrão } \\
( \pm \mathrm{R} \$)\end{array}$ \\
\hline Salários & 1.462 & $112(7,7)$ & $1.350(92,3)$ & 907,60 & $1.525,31$ \\
\hline Programas de Transf. de Renda & 1.659 & $1.187(71,5)$ & $472(28,5)$ & 74,94 & 46,08 \\
\hline Programa Bolsa Família (PBF) & 1.655 & $1.242(75,0)$ & $413(25,0)$ & 70,42 & 27,31 \\
\hline Outros Programas ${ }^{b}$ & 1.659 & $1.582(95,4)$ & $77(4,6)$ & 81,70 & 90,41 \\
\hline Seguro-Desemprego & 1.653 & $1.606(97,2)$ & $47(2,8)$ & 452,91 & 130,37 \\
\hline Aposentadoria & 1.647 & $1.494(90,7)$ & $153(9,3)$ & 522,00 & 387,54 \\
\hline Pensão Alimentícia & 1.658 & $1.578(95,2)$ & $80(4,8)$ & 291,15 & 509,47 \\
\hline Pensão (outra) & 1.656 & $1.600(96,6)$ & $56(3,4)$ & 518,25 & 578,04 \\
\hline Renda Mensal Vitalícia & 1.659 & $1.646(99,2)$ & $13(0,8)$ & 356,85 & 125,26 \\
\hline Aluguel & 1.656 & $1.636(98,8)$ & $20(1,2)$ & 557,50 & 921,94 \\
\hline Doações (Igrejas, ONGs, Instituições) & 1.658 & $1.654(99,8)$ & $4(0,2)$ & 63,75 & 34,73 \\
\hline Venda de produtos & 1.653 & $1.577(95,4)$ & $76(4,6)$ & 233,62 & 434,55 \\
\hline
\end{tabular}

${ }^{\mathrm{a}}$ Se inferior a 1.659, a diferença deve-se à inexistência das informações no banco de dados; ${ }^{\mathrm{b}}$ Programa Bolsa Escola, Benefício de prestador de contas (BPC), Auxílio Gás, Cartão Alimentação, Programa de Erradicação do Trabalho Infantil (PETI) e Programa Agente Jovem. 
a vulnerabilidade destas famílias para compra de alimentos.

As características demográficas da amostra estudada são apresentadas na Tabela 2. Observa-se que $29,4 \%(n=510)$ das crianças menores de 2 anos, pertenciam a famílias cadastradas em PTCR.

Entre as crianças que viviam na região urbana, 21,8\% pertenciam a famílias cadastradas em algum PTCR. Na região rural este número é significativamente maior, incluindo $33,5 \%$ das crianças ( $\mathrm{p}=0.0038)$. Apesar de, em números absolutos, a maior concentração de beneficiários residir em regiões urbanas, o que pode ser justificado pela grande concentração da população brasileira nas grandes cidades ${ }^{5}$, aproximadamente uma em cada três crianças da região rural é coberta por PTCR. Entre as crianças da região urbana, uma em cada cinco é beneficiária, sendo notória a maior vulnerabilidade das famílias que vivem na região rural.

Aproximadamente uma em cada três crianças da região Nordeste eram de famílias beneficiárias de PTCR ( $\mathrm{p}=0.0009)$. Outro estudo evidenciou que para $43,3 \%$ dos beneficiários do $\mathrm{PBF}$ residentes na região Nordeste, o valor do benefício representava $\geq 27,4 \%$ do total dos rendimentos financeiros da família, mostrando o alto grau de dependência financeira do valor transferido pelo programa entre as famílias desta região ${ }^{25}$.

Os dados brasileiros da Pesquisa de Orçamento familiar (POF) realizada entre 2008/2009 apontaram que a despesa média per capita dos $10 \%$ das famílias com os maiores rendimentos financeiros era 9,6 vezes a dos $40 \%$ com menores rendimentos, confirmando este abismo social que existe entre as famílias brasileiras. A região mais desigual foi a Nordeste com 11,3 vezes, e a menos

Tabela 2. Características demográficas, socioeconômicas e Segurança Alimentar de crianças menores de 2 anos, segundo o recebimento do benefício de Programa de Transferência Condicionada de Renda (PTCR), PNDS, Brasil, 2006. $(\mathrm{n}=1.735)$

\begin{tabular}{|c|c|c|c|c|}
\hline Variáveis & $\mathbf{n}^{\mathrm{a}}$ & $\begin{array}{c}\text { Beneficiárias PTCR }{ }^{\mathrm{b}} \\
\text { n }(\% / \mathrm{IC} 95 \%)\end{array}$ & $\begin{array}{c}\text { Não Beneficiárias PTCR }{ }^{\text {b }} \\
\text { n(\%/IC95\%) }\end{array}$ & $\begin{array}{c}\mathrm{p} \\
\chi^{2} \text { test }\end{array}$ \\
\hline Crianças de 0 a 2 anos & $1.735^{c}$ & $510(29,4 / 27,0-31,9)$ & $1.225(70,6 /(68,1-73,0)$ & \\
\hline Zona de moradia & & & & 0.0038 \\
\hline Urbano & 1.169 & $315(21,8 / 17,5-26,8)$ & $854(78,2 / 73,2-82,5)$ & \\
\hline Rural & 566 & $195(33,5 / 27,3-40,3)$ & $371(66,5 / 59,7-72,7)$ & \\
\hline Macrorregiões & & & & 0.0009 \\
\hline Norte & 409 & $138(26,7 / 21,9-32,2)$ & $271(73,3 / 67,8-78,1)$ & \\
\hline Nordeste & 350 & $146(36,2 / 29,5-43,5)$ & $204(63,8 / 56,5-70,5)$ & \\
\hline Sudeste & 323 & $76(17,0 / 10,42-26,4)$ & $247(83,0 / 73,6-89,6)$ & \\
\hline Sul & 301 & $72(18,6 / 12,7-26,3)$ & $229(81,4 / 73,7-87,3)$ & \\
\hline Centro-oeste & 352 & $78(19,5 / 14,5-25,8)$ & $274(80,5 / 74,2-85,5)$ & \\
\hline Segurança Alimentar e Nutricional & & & & 0.0000 \\
\hline Segurança Alimentar & 820 & $153(18,7 / 1,6-21,5)$ & $667(81,3 / 78,5-83,9)$ & \\
\hline Insegurança Alimentar Leve & 472 & $136(28,8 / 24,7-33,3)$ & $336(71,2 / 66,7-75,3)$ & \\
\hline Insegurança Alim. Moderada & 231 & $116(50,2 / 43,3-57,1)$ & $115(49,8 / 42,9-56,7)$ & \\
\hline Insegurança Alimentar Grave & 132 & $69(52,3 / 43,1-61,3)$ & $63(47,7 / 38,7-56,9)$ & \\
\hline $\mathrm{ABEP}^{\mathrm{d}}$ & & & & 0.0000 \\
\hline Classe A & 11 & $0(0,0)$ & $11(100,0)$ & \\
\hline Classe B & 113 & $1(0,9 / 0,1-6,0)$ & $112(99,1 / 93,9-99,9)$ & \\
\hline Classe C & 429 & $55(12,8 / 10,1-16,2)$ & $374(87,2 / 83,8-89,9)$ & \\
\hline Classe D & 659 & $225(34,1 / 30,6-37,9)$ & $434(65,9 / 62,1-69,4)$ & \\
\hline Classe E & 417 & $182(43,7 / 38,8-48,6)$ & $235(56,3 / 51,4-61,1)$ & \\
\hline Escolaridade materna & & & & 0.0000 \\
\hline $0-4$ anos de estudo & 363 & $169(46,6 / 41,0-52,2)$ & $194(53,4 / 47,8-59,0)$ & \\
\hline 5-8 anos de estudo & 617 & $219(35,5 / 31,5-39,7)$ & $398(64,5 / 60,3-68,5)$ & \\
\hline 9 anos ou mais anos de estudo & 755 & $122(16,2 / 13,5-19,2)$ & $633(83,8 / 80,8-86,5)$ & \\
\hline
\end{tabular}

a Se inferior a 1.735, a diferença deve-se à inexistência das informações no banco de dados; ${ }^{\mathrm{b}}$ Programas de transferência condicionada de renda; ${ }^{\mathrm{c}}$ Amostra representa população de 4.974 .767 crianças brasileiras de 0 a 2 anos, quando os dados são expandidos; ${ }^{\mathrm{d}}$ Associação Brasileira das Empresas de Pesquisa. 
desigual a Sul com 6,9 vezes, reforçando novamente a grande desigualdade da distribuição de renda por regiões brasileiras. No entanto, este valor é menor que há seis anos, sugerindo uma tendência de queda da desigualdade no país ${ }^{26}$.

O estudo do IBASE de 2008, incluindo somente os beneficiários do PBF em todo o território nacional, chegou a resultados similares. $\mathrm{O}$ benefício representou, em média, $16,6 \%$ da renda familiar, e 78,3\% das famílias residiam na região urbana, com maior concentração de domicílios cobertos em bolsões de pobreza e favelas. Entre os beneficiários, 49,9\% residiam no Nordeste, ou seja, aproximadamente 5 dos $11 \mathrm{mi}-$ lhões de beneficiários do PBF neste período ${ }^{27}$.

Como tema diretamente vinculado à pobreza, a alimentação saudável, acessível, de qualidade, em quantidade suficiente e de modo permanente é um direito humano básico, garantido na Lei Orgânica de Segurança Alimentar e Nutricional aprovada em $2006^{28}$. Desde a Declaração Universal dos Direitos Humanos de 1948, a alimentação passa a ser vista como um direito humano fundamental. No Brasil, tais discussões ganham força a partir do segundo milênio, por meio das conferências sobre Segurança Alimentar e Nutricional ${ }^{29}$.

Em várias regiões do globo, a problemática que envolve a insuficiência de renda entre as populações e as consequências desta desigualdade social, atinge questões em diversas áreas e níveis, verificando situações de insegurança alimentar. As concentrações de renda em vários países levam ao agravamento de problemas antigos como a fome, ainda não superada, visto que, segundo dados das Nações Unidas, 33 milhões de pessoas passam fome na América do Sul, apesar de ser uma das maiores regiões produtoras de alimentos do mundo ${ }^{30}$.

Observa-se também na Tabela 2 que entre as crianças que se encontravam em situação de SA apenas $18,7 \%$ pertenciam aos PTCR. Entre as crianças com situação de IAL, IAM e IAG, 28,8\%, $50,2 \%$ e $52,3 \%$, respectivamente, pertenciam aos PTCR. Assim, embora este não seja um critério de inclusão, priorizam-se nos PTCR famílias com algum grau de insegurança alimentar.

A vulnerabilidade à insegurança alimentar está associada principalmente à desigualdade social e à pobreza $^{31}$. Os PTCR têm um papel relevante no combate à fome e à insegurança alimentar da população ${ }^{32}$. No entanto, apesar da transferência do recurso financeiro dos programas, grande parte destas crianças avaliadas ainda estava em situação de insegurança alimentar no Brasil em 2006.
Outros estudos brasileiros também identificam níveis de insegurança alimentar entre aqueles que recebem algum benefício dos PTCR. A pesquisa do IBASE demonstrou que $54,8 \%$ das famílias beneficiárias estavam em situação de insegurança alimentar moderada e grave $e^{27}$. Dados da Pesquisa Nacional por Amostra de Domicílios (PNAD), em 2004, mostraram que 66\% dos domicílios com beneficiários de PTCR encontravam-se em algum nível de insegurança alimentar leve, moderado ou grave ${ }^{33}$. Inquérito realizado no município de Toledo (PR), entre 2006 e 2007, evidenciou que $75,0 \%$ dos beneficiários do $\mathrm{PBF}$ sofriam com IA em diferentes níveis, sendo mais prevalente em domicílios com crianças e adolescentes $^{31}$. Tais resultados estão em consonância com o resultado significativo encontrado no presente trabalho em relação a esta variável, já que foram incluídas apenas crianças na amostra.

Por outro lado, entre as crianças em IAM e IAG, $49,8 \%$ e $47,7 \%$, respectivamente, não pertencem a qualquer PTCR $(p=0.0000)$, conforme demonstrado na Tabela 2. Apesar de a cobertura ser ampla, tais programas ainda não alcançavam grande parte das crianças brasileiras que passavam por restrições quantitativas de alimentos, podendo ter vivido a experiência de fome. Ou seja, como um dos objetivos destes programas é a garantia da segurança alimentar, um número significativo de crianças que deveriam receber PTCR não eram beneficiadas, sugerindo algumas falhas nos mecanismos de seleção, o que pode apontar erros de exclusão ou insuficiência do potencial de cobertura dos PTCR naquele período.

Quando comparado a outros programas sociais, os PTCR apresentam alguns aspectos positivos como baixo custo operacional, fortalecimento da economia local e autonomia dos usuários na utilização do recurso. Por outro lado, são pontos negativos o fato dos valores transferidos não serem suficientes para atender as necessidades básicas da família e a sua desvalorização, devido ao processo inflacionário. Também é comum os beneficiários realizarem compras a prazo em pequenos estabelecimentos que praticam preços mais altos. Por fim, a transferência do recurso dos PTCR para um grande número de famílias em pequenas comunidades leva ao aumento da demanda e, consequentemente, ao aumento dos preços dos alimentos em nível local ${ }^{4}$.

$\mathrm{O}$ foco dos programas em atingir aqueles com piores condições econômicas estava sendo cumprido em 2006. Entre as famílias de crianças menores de 2 anos com alto poder de compra (classe A) não havia beneficiários dos PTCR. Entre as 
famílias de crianças com menor poder de compra, $34,1 \%$ e $43,7 \%$ (classe D e E respectivamente), pertenciam aos PTCR $(p=0.0000)$. Os critérios utilizados na $\mathrm{ABEP}$ permitem dimensionar a capacidade de consumo de bens duráveis que aquela família conseguiu adquirir nos últimos anos, associados a dados como escolaridade do chefe da família e número de empregados mensalistas. Sendo assim, esta variável permite conhecer de forma mais ampla, a situação econômica das famílias, não apenas no momento da pesquisa, mas como se desenvolveu ao longo de um determinado tempo ${ }^{18}$.

A população de maior vulnerabilidade tem características específicas, sendo a escolaridade um fator diretamente associado ao risco social. Conforme observado na Tabela 2, quanto maior o número de anos de estudo da mãe, que é considerada aquela que assume o papel de cuidadora e responsável pelo bem estar de toda a família, menor a prevalência de famílias beneficiárias de PTCR. Entre as mães que estudaram entre 0 e 4 anos, $46,6 \%$ pertencem a famílias beneficiadas por algum dos programas, e entre aquelas que estudaram mais de 8 anos, apenas $16,2 \%$ pertenciam aos PTCR $(\mathrm{p}=0.0000)$. Vale ressaltar que a maioria dos PTCR na América Latina tende a privilegiar as mulheres na titularidade do benefício, sendo no caso do PBF, 93,6\% dos titulares do sexo feminino ${ }^{27}$.

A baixa renda per capita em algumas macrorregiões brasileiras, como é o caso das regiões Norte e Nordeste, sofre alguma influência da concentração de indivíduos com baixa escolaridade $^{34}$. Um número expressivo da população brasileira vive à margem da sociedade, não conseguindo sair da situação de vulnerabilidade.

Os PTCR têm entre seus eixos principais as condicionalidades, que devem ser cumpridas pelos beneficiários, para que continuem recebendo o recurso. No caso do PBF tais condições têm por objetivo garantir o acesso aos direitos sociais básicos, nas áreas de educação, saúde e assistência social. $\mathrm{Na}$ área da saúde as famílias assumem o compromisso de acompanhar o cartão de vacinação e o crescimento e desenvolvimento das crianças menores de 7 anos9.

Conhecer o público beneficiado é fundamental para avaliar se os objetivos estão sendo atingi$\operatorname{dos}^{35}$. Para as variáveis nutricionais, que traduzem a condição de saúde das crianças brasileiras menores de 2 anos, evidenciou-se que $28,7 \%$ das que apresentaram peso adequado para a idade eram beneficiária de PTCR. Entre os desvios nutricionais prevaleceu, em números absolutos, o peso elevado para a idade $(\mathrm{n}=34)$ em relação ao baixo peso para a idade $(n=20)$, apesar de não apresentar significância estatística para esta análise.

A desnutrição no Brasil era considerada um dos maiores desafios da saúde pública do país, mas esta realidade vem apresentando mudanças. Dados de 419 municípios, de 23 estados brasileiros, da pesquisa nutricional de crianças residentes em assentamentos e regiões semiáridas do país, demonstraram a prevalência de baixo peso para a idade em crianças menores de cinco anos de $5,5 \%$ para aqueles pertencentes ao PBF, e 4,0\% para aqueles que não estão expostos ao programa, indicando prevalências semelhantes entre os dois grupos ${ }^{36}$. Os dados da PNDS de 1996 e 2006 apresentaram tendência de declínio para o déficit de peso para a idade entre as crianças brasileiras de 0 a 5 anos, principalmente nos estratos de menor poder aquisitivo ${ }^{37}$.

O processo de transição nutricional, caracterizado pela mudança no padrão de distribuição dos distúrbios nutricionais, em que há queda da prevalência de desnutrição e um aumento expressivo do excesso de peso e obesidade, parece estar presente entre grande parte dos brasileiros, principalmente na população de maior vulnerabilidade social ${ }^{38,39}$.

Avaliando dados da pesquisa do IBASE, com beneficiários do PBF, foi identificado o aumento do consumo de alimentos de todos os grupos a partir do recebimento do benefício. Isto poderia sugerir um efeito positivo do incremento da renda destas famílias pelo programa. No entanto, entre os que viviam em IAG e as famílias que mais dependiam do valor do recurso devido à baixa renda, houve aumento do consumo de alimentos com alta densidade energética como os processados, o açúcar e os refrigerantes. O maior poder de compra das famílias mais pobres parece aumentar as escolhas de alimentos não saudáveis ${ }^{25}$.

A grande mudança no estilo de vida estimula o sedentarismo e os maus hábitos alimentares entre as crianças de todas as classes sociais que ocupam grande parte do tempo assistindo televisão. Isto diminui significativamente o tempo das crianças gasto com atividade física e aumenta o risco de serem influenciados pela indústria de alimentos, por meio da publicidade, para consumir produtos industrializados com excesso de energia, açúcares, gorduras e sal em substituição aos alimentos caseiros ou naturais ${ }^{40,41}$. Todos estes elementos compõem um ambiente propício para o desenvolvimento de doenças crônicas não transmissíveis, trazendo prejuízos tanto à família quanto à sociedade. 
Os dados referentes ao tempo AME e, consequentemente, à introdução de alimentos em idade precoce (Tabela 3), demonstram a oferta de quaisquer líquidos, semissólidos e sólidos antes de seis meses de vida entre os dois grupos, representando dados preocupantes em relação à saúde das crianças analisadas neste estudo. Muitas delas receberam precocemente a oferta de alimento que não o leite materno antes dos seis meses de vida, sendo estas crianças em parte pertencentes a famílias beneficiárias de PTCR, ou seja, com maior vulnerabilidade social. Entre as crianças que receberam aleitamento materno exclusivo apenas até o segundo mês de vida, $31,2 \%$ pertencia aos PTCR $(\mathrm{p}=0.0304)$. Além de não haver vantagens em se iniciar os alimentos complementares antes dos seis meses, podendo trazer prejuízos à saúde da criança como o aumento de episódios de diarreia e infecção respiratória, esta introdução precoce expõem tais crianças ao risco de desenvolvimento de distúrbios nutricionais, como o excesso de peso ou a desnutrição $0^{42}$.

Um estudo realizado com 270 crianças frequentadoras de creches públicas no Município de São Paulo demonstrou que $2 / 3$ das crianças recebiam alimentos com potencial obesogênico antes dos 12 meses de vida, sendo os filhos de mães com baixa escolaridade, mais jovens e com menor renda, os mais susceptíveis ao erro alimentar da introdução precoce de alimentos industrializados ${ }^{40}$. Além destes riscos, este fato aumenta os gastos da família, que já tem comprometimento da renda. O uso de fórmula infantil pode representar custo 3,3 vezes, e o uso do leite de vaca 1,7 vezes maior que o custo do aleitamento materno ${ }^{43}$.

\section{Conclusão}

A maior parte das crianças menores de 2 anos de famílias beneficiárias dos PTCR em 2006 apresentavam características correspondentes à população mais vulnerável, como residir em regiões mais pobres do país, ter menor renda familiar, ter menor poder compra, ter baixa escolaridade materna, estar em insegurança alimentar, atingindo os grupos populacionais para os quais foram criados.

Sendo a transferência do recurso uma forma de tentar garantir a segurança alimentar e considerando a pobreza um fenômeno multidimensional, o valor do benefício parecia não ser suficiente para garantir a efetividade da intervenção, já que se mantinham níveis de insegurança alimentar em grandes proporções nos domicílios dos beneficiários. Além disso, entre os indivíduos em IA moderada e grave, grande parte não recebia qualquer benefício destes programas, sugerindo que a cobertura neste período não era suficiente para atingir a todos que deles necessitavam.

O maior número de crianças menores de 2 anos com excesso de peso entre os desvios nutricionais parece acompanhar o fenômeno da transição nutricional. Sugere-se que novas pesquisas busquem compreender as escolhas alimentares das famílias que recebem os PTCR a fim de indicar um caminho para a formulação de condicionalidades mais efetivas.

A forte tendência da introdução precoce de alimentos antes dos seis meses de vida entre as crianças menores de 2 anos beneficiárias de PTCR demostrou a necessidade de intervenções educacionais relacionadas à saúde e à alimentação de

Tabela 3. Caracterização das variáveis nutricionais de crianças menores de 2 anos, segundo o recebimento do benefício de Programa de Transferência Condicionada de Renda (PTCR), PNDS, Brasil, 2006. ( $\mathrm{n}=$ 1.735)

\begin{tabular}{|c|c|c|c|c|}
\hline Variáveis & $\mathbf{n}^{\mathrm{a}}$ & $\begin{array}{c}\text { Beneficiárias } \text { PTCR }^{\mathrm{b}} \\
\text { n }(\% / \mathrm{IC} 95 \%)\end{array}$ & $\begin{array}{c}\text { Não Beneficiárias PTCR } \\
\text { n(\%/IC95\%) }\end{array}$ & $\begin{array}{c}\mathbf{p} \\
\chi^{2} \text { test }\end{array}$ \\
\hline Estado Nutricional (P/I) & & & & 0.1271 \\
\hline Baixo peso para idade & 51 & $20(39,2 / 26,3-53,8)$ & $31(60,8 / 46,2-73,7)$ & \\
\hline Peso adequado para idade & 1.588 & $456(28,7 / 26,3-31,3)$ & $1.132(71,3 / 68,7-73,7)$ & \\
\hline Peso elevado para idade & 96 & $34(35,4 / 26,4-45,7)$ & $62(64,6 / 54,3-73,7)$ & \\
\hline Tempo de $\mathrm{AME}^{\mathrm{c}}$ & & & & 0.0304 \\
\hline De 0 a 2 meses & 873 & $272(31.2 / 27.8-34.7)$ & $601(68.8 / 65.3-72.2)$ & \\
\hline Entre 2 a 4 meses & 331 & $77(23.3 / 18.9-28.3)$ & $254(76.7 / 71.7-81.1)$ & \\
\hline Entre 4 a 6 meses & 409 & $123(30.1 / 25.7-34.8)$ & $286(69.9 / 65.2-74.3)$ & \\
\hline
\end{tabular}

a Se inferior à 1.735, a diferença deve-se à inexistência das informações no banco de dados; ${ }^{\mathrm{b}}$ Programas de transferência condicionada de renda; ${ }^{c} A M E$ : Aleitamento Materno Exclusivo. 
forma mais eficiente, integrando a transferência de renda às ações que possibilitem a promoção da saúde e a prevenção de doenças crônicas não transmissíveis.

\section{Colaboradores}

JB Vega e JAAC Taddei participaram da concepção, delineamento, análise e interpretação dos dados, redação e revisão crítica do artigo, e aprovação da versão a ser publicada. AP Poblacion participou da concepção, delineamento, redação e revisão crítica do artigo, e aprovação da versão a ser publicada.

\section{Referências}

1. Ferreira MAF, Latorre MRDO. Desigualdade social e os estudos epidemiológicos: uma reflexão. Cien Saude Colet 2012; 17(9):2523-2531.

2. Monnert GL, Senna MCM, Schottz V, Magalhães R, Burlandy L. Do direito incondicional à condicionalidade do direito: as contrapartidas do Programa Bolsa Família. Cien Saude Colet 2007; 12(6):1453-1462.

3. Neri M, Soares W. Desigualdade social e saúde no Brasil. Cad Saude Publica 2002; 18(Supl.):77-87.

4. Burlandy L. Transferência condicionada de renda e segurança alimentar e nutricional. Cien Saude Colet 2007; 12(6):1441-1451.

5. Instituto Brasileiro de Geografia e Estatística (IBGE). Estudo e Pesquisa. Informações demográficas $n^{\circ} 27$. Síntese de indicadores sociais. Uma análise das condições de vida da população brasileira. Rio de Janeiro: IBGE; 2010.

6. Zimmermann CR. Os programas sociais sob a ótica dos direitos humanos: o caso do Bolsa Família do governo Lula no Brasil. Sur, Rev. int. direitos human 2006; 3(4):144-159.

7. Bichir RM. O Bolsa Família na berlinda? Os desafios atuais dos programas de transferência de renda. Novos estu. - CEBRAP 2010; 87:115-129.

8. Silva MOS. O Bolsa Família: problematizando questões centrais na política de transferência de renda no Brasil. Cien Saude Colet 2007; 12(6):1429-1439.

9. Brasil. Ministério do Desenvolvimento Social (MDS) [Internet]. Brasil: Programa Bolsa Família. [acessado 2012 out 2]. Disponível em: http://www.mds. gov.br/bolsafamilia

10. Oliveira FCC, Cotta RMM, Sant'Ana LFR, Priore SE, Franceschini SCC. Programas Bolsa Família e estado nutricional infantil: desafios estratégicos. Cien Saude Colet 2011; 16(7):3307-3316.

11. Campbell CC. Food insecurity: a nutritional outcome or a predictor variable? J Nutr. 1991; 121(3): 408-415.

12. Ministério da Saúde (MS). Pesquisa Nacional de Demografia e Saúde da Criança e da Mulher (PNDS 2006) [Internet]. Brasil: MS; [2007]. [acessado 2012 maio 12]. Disponível em: http://bvsms.saude.gov.br/ bvs/pnds 
13. Cavenaghi S. Aspectos metodológicos e comparabilidade com pesquisas anteriores. In: Ministério da Saúde (MS). Centro Brasileiro de Análise e Planejamento. Pesquisa Nacional de Demografia e Saúde da Criança e da Mulher (PNDS 2006). Dimensões do Processo Reprodutivo e da Saúde da Criança. Série G. Estatística e informação em saúde. Brasília: MS; 2009. p. 14-32.

14. Habicht JP, Yarbrough C, Martorell R, Malina RM, Klein R. Height and weight standards for preschool children. How relevant are ethnics differences in growth potencial? Lancet 1974; 1(7858):661-664.

15. Barros FC, Victora CG, Scherpbier R, Gwatkin D. Socioeconomic inequities in the health and nutrition of children in low/middle income countries. Rev Saude Publica 2010; 44(1):1-16.

16. Segall-Corrêa AM, Marín-León L. A segurança alimentar no Brasil: proposição e usos da Escala Brasileira de Medida de Insegurança Alimentar (EBIA) de 2003 a 2009. Segurança Alimentar e Nutricional 2009; 16(2):1-19.

17. Corrêa AMS, Marín-León L, Panigassi G, PérezEscamilla R. Segurança alimentar no domicílio. In: Ministério da Saúde (MS) Centro Brasileiro de Análise e Planejamento. Pesquisa Nacional de Demografia e Saúde da Criança e da Mulher (PNDS 2006). Dimensões do Processo Reprodutivo e da Saúde da Criança. Brasília: MS; 2009. p. 231-248. (Série G. Estatística e informação em saúde)

18. Associação Brasileira de Empresas e Pesquisa (ABEP). Critério de Classificação Econômica Brasil (CCEB) [Internet]. São Paulo: ABEP; versão 2008 [acesso 2012 ago 20]. Disponível em: http://www. abep.org/novo/Content.aspx?ContentID = 139 .

19. World Health Organization (WHO). Child growth standards: length/height-for-age, weight-for-age, weightfor- lenght, weight-fot-height and body mass index-for-age: methods and development. Geneve: WHO; 2006.
20. Corrêa AMS, Marín-León L, Panigassi G, Rea MF Pérez-Escamilla R. Amamentação e alimentação infantil. In: Ministério da Saúde (MS) Centro Brasileiro de Análise e Planejamento. Pesquisa Nacional de Demografia e Saúde da Criança e da Mulher (PNDS 2006). Dimensões do Processo Reprodutivo e da Saúde da Criança. Brasília: MS; 2009. p.196-212. (Série G. Estatística e informação em saúde)

21. Silva MOS. Pobreza, desigualdade e políticas públicas: caracterizando e problematizando a realidade brasileira. Rev. Katál. Florianópolis 2010; 13(2): 155-163.

22. Instituto de Pesquisa Econômica Aplicada (Ipea). A Década Inclusiva (2001-2011): Desigualdade, Pobreza e Políticas de Renda. Brasília: Ipea; 2012. Comunicado n.155.

23. Brasil. Lei $n^{\circ} 11.321$, de 7 de julho de 2006. Dispõe sobre o salário mínimo a partir de $1^{\circ}$ de abril de 2006. Diário Oficial da União 2006; 10 jul.

24. Fundação de Proteção e Defesa do Consumidor (PROCON). Pesquisas de cestas básicas anuais. Relatório anual de 2006 [Internet]. São Paulo: Equipe de Pesquisas - DEP - PROCON; 2006. [acessado 2012 nov 3]. Disponível em: http://www.procon.sp. gov.br/categoria.asp?id $=551$

25. Lignani JB, Sichieri R, Burlandy L, Salles-Costa R. Changes in food consumption among the Programa Bolsa Família participant families in Brazil. Pub Health Nutr 2010; 14(5):785-792.

26. Instituto Brasileiro de Geografia e Estatística (IBGE). Pesquisa de Orçamentos Familiares 2008/2009. Despesas, rendimentos e condições de vida [Internet]. Rio de Janeiro: IBGE; [página na Internet]. 2009. [acessado 2012 nov7]. Disponível em: http://www. ibge.gov.br/home/estatistica/populacao/condicao devida/pof/2008_2009/POFpublicacao.pdf

27. Instituto Brasileiro de Análises Sociais e Econômicas (IBASE). Repercussões do programa bolsa família na segurança alimentar e nutricional. Relatório-Síntese. Rio de Janeiro: IBASE; 2008. 
28. Brasil. Lei $\mathrm{n}^{\circ} 11.346$ de 15 de setembro de 2006. Cria o Sistema Nacional de Segurança Alimentar e Nutricional - SISAN com vistas em assegurar o direito humano à alimentação adequada e dá outras providências. Diário Oficial da União 2006; 15 set.

29. Ramos CI, Cuervo MRM. Programa Bolsa Família: a interface entre a atuação profissional e o direito humano a alimentação adequada. Cien Saude Colet 2012; 17(8):2159-2168.

30. Organización de las Naciones Unidas para La Alimentación y La Agricultura (FAO). Oficina Regional de la FAO para América Latina y el Caribe [Internet]. Santiago: FAO; 2012. [página na Internet]. [acessado $2012 \mathrm{dez} 2$ ]. Disponível em: http://www. rlc.fao.org

31. Anschau FR, Matsuo T, Segall-Corrêa AM. Insegurança alimentar entre beneficiários de programas de transferência de renda. Rev. Nutr. 2012; 25(2):177189.

32. Salles-Costa R, Pereira RA, Vasconcellos MTL, Veiga GV, Marins VMR, Jardim BC, Gomes FS, Sichieri R. Associação entre fatores socioeconômicos e insegurança alimentar: estudo de base populacional na Região Metropolitana do Rio de Janeiro, Brasil. Rev. Nutr. 2008; 21(Supl.):99s-109s.

33. Instituto Brasileiro de Geografia e Estatística (IBGE). Pesquisa Nacional por Amostra de Domicílios. Aspectos complementares de educação e acesso a transferências de renda de Programas sociais [Internet]. Rio de Janeiro: IBGE; 2006. [acessado 2012 out 20]. Disponível em: http://www.ibge.gov.br/ home/presidencia/noticias/noticia_impressao.php? id_noticia $=562$

34. Salvato MA, Ferreira PCG, Duarte AJM. O impacto da escolaridade sobre a distribuição de renda. Estud. Econ. 2010; 40(4):753-791.

35. Medeiros M, Britto T, Soares F. Transferência de renda no Brasil. Novos Estudos 2007; 79:5-21.

36. Paes-Sousa R, Santos LMP, Miazaki ES. Effects of a conditional cash transfer programme on child nutrition in Brazil. Bull World Health Organ 2011; 89(7):496-503.
37. Monteiro CA, Conde WL, Konno SC, Lima ALL, Silva ACF, Benicio MHD. Avaliação antropométrica do estado nutricional de mulheres em idade fértil e crianças menores de cinco anos. In: Ministério da Saúde (MS). Centro Brasileiro de Análise e Planejamento. Pesquisa Nacional de Demografia e Saúde da Criança e da Mulher (PNDS 2006). Dimensões do Processo Reprodutivo e da Saúde da Criança. Brasília: MS; 2009. p. 214-230.(Série G. Estatística e informação em saúde)

38. Silva DA. Sobrepeso e obesidade em crianças de cinco a dez anos de idade beneficiárias do programa bolsa família no estado de Sergipe, Brasil. Rev. Paul Pediatr 2011; 29(4):529-535.

39. Mondini L, Gimeno SGA. Transição Nutricional: Significado, Determinantes e Prognósticos. In: Taddei JA, Lang RMF, Longo-Silva G, Toloni MHA, organizadores. Nutrição e Saúde Pública. Rio de Janeiro: Rubio; 2011. p. 561-575.

40. Toloni MHA, Longo-Silva G, Goulart RMM, Taddei JAAC. Introdução de alimentos industrializados e de alimentos de uso tradicional na dieta de crianças de creches públicas no município de São Paulo. Rev. Nutr. 2011; 24(1):61-70.

41. Sichieri R, Souza RA. Estratégias para prevenção da obesidade em crianças e adolescentes. Cad Saude Publica 2008; 24(2):S209-S234.

42. Brasil. Ministério da Saúde (MS). Secretaria de Atenção à Saúde Departamento de Atenção Básica Saúde da Criança. Nutrição Infantil Aleitamento Materno e Alimentação Complementar. Brasília: MS; 2009. (Série A. Normas e Manuais Técnicos Cadernos de Atenção Básica, n. ${ }^{\circ} 23$ ).

43. Barbosa MB, Palma D, Bataglin T, Taddei JAAC. Custo da alimentação no primeiro ano de vida. Rev. Nutr. 2007; 20(1):55-62.

Artigo apresentado em 28/03/2013

Aprovado em 23/06/2013

Versão final apresentada em 01/07/2013 